\title{
Recently Emerging Signaling Landscape of Ataxia-Telangiectasia Mutated (ATM) Kinase
}

\author{
Ammad Ahmad Farooqi ${ }^{1 *}$, Rukset Attar ${ }^{2}$, Belkis Atasever Arslan ${ }^{3}$, Mirna Azalea \\ Romero $^{4}$, Muhammad Fahim ul Haq ${ }^{5}$, Muhammad Imran Qadir ${ }^{6}$
}

\begin{abstract}
Research over the years has progressively and sequentially provided near complete resolution of regulators of the DNA repair pathways which are so important for cancer prevention. Ataxia-telangiectasia mutated kinase (ATM), a high-molecular-weight PI3K-family kinase has emerged as a master regulator of DNA damage signaling and extensive cross-talk between ATM and downstream proteins forms an interlaced signaling network. There is rapidly growing scientific evidence emphasizing newly emerging paradigms in ATM biology. In this review, we provide latest information regarding how oxidative stress induced activation of ATM can be utilized as a therapeutic target in different cancer cell lines and in xenografted mice. Moreover, crosstalk between autophagy and ATM is also discussed with focus on how autophagy inhibition induces apoptosis in cancer cells.
\end{abstract}

Keywords: Ataxia-telangiectasia mutated kinase - signalling landscape - cancer therapy

Asian Pac J Cancer Prev, 15 (16), 6485-6488

\section{Introduction}

Research over decades has gradually and sequentially provided near complete resolution of DNA damage signaling and it is now known that DNA damage repair pathway consists of protein network that is highly branched, tightly regulated and well orchestrated. Ataxiatelangiectasia mutated kinase (ATM), ATR and DNAPKcs are members of phosphoinositide-3-kinase-related protein kinase (PIKK) family. It is evident that DNA damage mediated activation of PIKK family members ATM, ATR and DNA-PKcs is further systematically categorized into modes of repair including homologous recombination (HR) and non-Homologous End Joining (NHEJ).

Emerging lines of evidence reveal that ATM is a master regulator of DNA damage signaling and gatekeeper of genome integrity. It has lately been shown that ATM-mediated mitotic arrest-deficient protein 1 (Mad1) phosphorylation at Serine 214 induced homodimerization of Mad1 and its heterodimerization with Mad2. These mechanisms were noted to be necessary for chromosomal stability (Yang et al., 2014). Hepatocyte nuclear factor-1 alpha $(\mathrm{HNF} 1 \alpha)$ is also phosphorylated by ATM at Ser249 (Zhao et al., 2014). Phosphorylation at serine 403 of HDMX has been shown to facilitate binding of
C-terminal RING domain of HDMX to the nascent p53 mRNA to trigger p53 synthesis (Malbert-Colas et al., 2014). Increasingly it is being realized that adhesion between cancer cells and extracellular matrix (ECM) proteins, is an initial step of metastasis. In-vitro evidence has shown that surface expression of $\alpha 5 \beta 1$ integrin was increased in the cells treated with ionizing radiation. In MDA-MB-231 cells, it has been reported that $\alpha 5 \beta 1$ integrin surface expression was considerably reduced upon ATM inhibition (Lee et al., 2014). Surprisingly it has been shown that caspase-3 processed Cdc6 induced activation of ATM in HeLa cells (Liu et al., 2014). SETD2 is a histone methyltransferase reported to be involved in activation of ATM. In-vitro analysis indicated that SETD2 silenced cells did not show activated ATM kinase (Carvalho et al., 2014). There is an intriguing piece of experimental evidence suggesting that recombinant lysyl oxidase propeptide rLOX-PP considerably reduced radiation induced ATM activation in DU145 and PC3 cells. Likewise, rLOX-PP significantly reduced tumor growth in mice xenografted with cancer cells (Bais et al., 2014). ATM has been shown to phosphorylate deleted in breast cancer-1 (DBC-1) to increase its binding affinity with deacetylase SIRT1. DBC-1 bound SIRT1 was unable to deacetylate $\mathrm{p} 53$ thus p53 triggered expression of its target genes. Protein expression of SIRT1 is overexpressed in

${ }^{\prime}$ Laboratory for Translational Oncology and Personalized Medicine, Rashid Latif Medical College, ${ }^{5}$ Department of Biochemistry, RLMC, Lahore, Pakistan, ${ }^{2}$ Department of Obstetrics and Gynecology, Yeditepe University Faculty of Medicine, ${ }^{3}$ Department of Molecular Biology and Genetics, Faculty of Engineering and Natural Sciences, Üsküdar University, Istanbul, Turkey, ${ }^{4}$ Laboratorio de Farmacología, Unidad Académica de Medicina, Universidad Autónoma de Guerrero, Av. Solidaridad S/N Col. Hornos Insurgentes, Acapulco Guerrero México, ${ }^{6}$ Institute of Molecular Biology and Biotechnology, BZU, Multan *For correspondence: ammadahmad638@yahoo.com 
FLT3-ITD+-cells and regulated by FLT3 kinase activity. Genetic or chemical inhibition of SIRT1 remarkably enhanced p53 mediated apoptosis (Sasca et al., 2014). It has recently been persuasively revealed that ATM was not induced in irradiated HER2 silenced MCF-7 breast cancer cells (Yan et al., 2014).

\section{Oxidative Stress Induced Activation of ATM}

Elevated levels of ROS and persistent DNA damage are hallmark features of stress induced-premature senescence. Persistent DNA damage is indicative of prolonged ATM activity and wide ranging proteins. Recently emerging evidence is providing an overview of intricate protein network in senescent cells. ATM kinase regulates different proteins in senescent cells. Treating Human endometriumderived mesenchymal stem cells (hMESCs) with $\mathrm{H} 2 \mathrm{O} 2$ induced ROS generation that consequently stimulated ATM kinase activity. Considerably enhanced activity of p38MAPK/MAPKAPK-2 was noted in $\mathrm{H} 2 \mathrm{O} 2$ treated cells. Interestingly, there was a persistent DNA damage response and ROS generation noted in $\mathrm{H} 2 \mathrm{O} 2$ treated cells. p38MAPK activity modulated prolonged DDR and ROS generation, as cells treated with pharmacological inhibitors of p38MAPK displayed remarkably reduced ROS generation (Borodkina et al., 2014). Moreover, Insulin like Growth Factor (IGF-1) induced intracellular signaling stimulated expression of Secretory clusterin (sCLU) via utilizing MAPK/ERK-1/2/Egr-1 pathway. Functionally inactive ATM expressing fibroblasts have lower expression of sCLU, thus suggesting that ATM also regulates its expression (Luo et al., 2014). Chaetocin, a histone methyltransferase inhibitor has also been noted to trigger ROS mediated ATM activation in glioma cells. Moreover, ATM was involved in induction of apoptosis in glioma cells via different mechanisms. ATM considerably enhanced interaction of p73, p300 and Yes-associated protein 1 (YAP1) in glioma cells. Additionally ATM functionalized intrinsic pathway via activation of Caspase-9. Conversely ATM mediated Caspase-9 activation was impaired upon treatment with ATM inhibitor (Dixit et al., 2014).

\section{Resveratrol Mediated ATM Activation}

Certain derivatives of BMH-21, a heterocyclic small molecule triggered DSB induced ATM activation (Colis et al., 2014). Previous studies have shown that Resveratrol induced activation of ATM via DSBs or enhancing generation of ROS. However, it has recently been shown that treating cells with DNA damaging agents or $\mathrm{H}_{2} \mathrm{O}_{2}$ induced ATM activation, however, these responses were attenuated upon treatment with ATM inhibitors. It was reported that Resveratrol mediated activation of ATM through oxidation. As it is already known that there is a formation of disulfide bonds between ATM monomers, therefore, disulfide-specific reducing agent TCEP was used to note if Resveratrol mediated activation of ATM was impaired. The results revealed notably reduced Resveratrol induced ATM activation in TCEP treated cells (Lee et al., 2014).

\section{Autophagy: Introduction}

Autophagy is a biologically complex cytoprotective mechanism. There is an overwhelmingly increasing list of research emphasizing on molecular machinery involved in induction of autophagy. It has been revealed that Atg8 is small ubiquitin (Ub)-like protein noted to be conjugated to autophagic membranes. Atg8 also conjugates different other Ub-like proteins including Atg12-Atg 5 to membranes by interacting with Atg 12 through its Atg8-interacting motif (AIM). Atg1, Atg13 and Atg 17 assembled to form functionally active Atg1. Vesicle nucleation is an intricate step triggered by Atg6 and Vps34 and Atg7 and Atg3 modulate vesicle elongation. Autophagy is a deeply studied mechanism as a response to chemotherapeutic treatment in cancer cells (Marino et al., 2014; Pandey and Chandravati, 2012).

\section{Synthetic and Natural agents mediated regulation of autophagy}

Earlier it has been reported that calpain processed Atg5 acted as a pro-apoptotic mitochondrion-permeabilizing fragment. Using papain-like cysteine protease inhibitor (E64d) it was noted that calpain-mediated Atg5 cleavage was remarkably repressed. Atg5 cleavage was also not observed in calpain silenced cells (Yousefi et al., 2006). There is a direct piece of evidence suggesting role of Trichokonin, an antimicrobial peptide in modulating autophagy via calpain-mediated Bax and Atg5 cleavage that induced release of cytochrome c (Shi et al., 2013). Interestingly, Trichokonin triggered ROS generation that triggered disposal of damaged mitochondria within autophagosomes via Atg5-mediated and mitochondriaselective autophagy (Shi et al., 2013). Arnica Montana and Arnica chamissonis derived helenalin has been shown to exert its effects via inhibition of NF- $x \mathrm{~B}$ p65 in MCF-7. Overexpressing p65 in MCF-7 dramatically abrogated helenalin induced autophagy (Lim et al., 2012).

\section{Interplay of ATM and Autophagy}

LY2603618 is a Chk1 inhibitor reported to be an inducer of DDR in lung cancer cells. There was an increase in pATM levels in LY2603618 treated lung cancer cells. Surprisingly, autophagy was also induced in LY2603618 treated cancer cells and LY2603618 mediated inhibitory effects on cell proliferation were further enhanced upon treating cancer cells with autophagy inhibitors (Wang et al., 2014). It is also important to mention that autophagy was induced in colorectal cancer cells treated with low dose of camptothecin. Moreover, ATM-Chk2-p53-p21 pathway was also activated in cancer cells that resulted in induction of premature senescence. Autophagy inhibitors considerably enhanced apoptosis in camptothecin treated cancer cells (Zhang et al., 2014). Likewise, Cisplatin induced autophagy in A549 cells as evidenced by higher pATM levels and pAMPK in drug treated cancer cells. Treating cancer cells with ATM inhibitors and autophagy inhibitors resulted in radiosensitization of A549 cells (Toulany et al., 2014). There is contemporary evidence 
indicating that ATM triggered activation of AMPK-ULK1 in U87MG and U251 glioma cell lines. Temozolomide induced apoptosis in glioma cell lines treated with ATM inhibitors and autophagy inhibitors (Zou et al., 2014). ATM inhibition with KU55933 induced autophagy in head and neck cancer cells. However, treating cancer cells with autophagy inducers dramatically enhanced KU55933 induced apoptosis (Lin et al., 2012).

It is now known that LKB1/AMPK/mTOR signaling axis controls autophagic induction (Han et al., 2013).

There is an exciting piece of evidence emphasizing on ATM mediated suppression of a negative regulator of autophagy, mTORC1. ATM involvement as an autophagy inducer was further confirmed in ATM-deficient MEFs, in which Nitric Oxide mediated autophagy induction was considerably impaired. ATM activated LKB via its phosphorylation and consequently, AMPK was activated by LKB. This pathway was verified in LKB1-deficient HeLa cells in which ATM was unable to transduce the signals to downstream AMPK because of absence of LKB (Tripathi et al., 2013).

Chemotherapeutic drugs mediated autophagic response in cancer cells is a stumbling block that needs to be overcome. Treating gastric cancer cell line SGC7901 with autophagy inhibitor, significantly enhanced cisplatin induced apoptosis (Zhang et al., 2013). Likewise, treating U251 and U87MG glioblastoma cells with autophagy inhibitors substantially enhanced gambogic acid induced apoptosis (Luo et al., 2012).

\section{Natural Agents Mediated ATM Activation}

G19, a sulfated oligosaccharide, prepared from Grateloupia filicina induced oxidative stress mediated activation of ATM in U-87 MG human glioma cells. More importantly, G19 was noted to effectively inhibit tumor growth in mice xenografted with U-87 MG tumor cells (Liu et al., 2014). Riccardin D, a macrocyclic bisbibenzyl, exerts its biological effects via inhibition of telomerase activity in MCF-7 and MDA-MB-231 breast cancer cells. Inhibition of telomerase activity has been shown to activate ATM, that consequently results in induction of apoptosis (Sun et al., 2014). Bufalin, an essential ingredient of Chinese Medicine also induced ATM expression in NCI-H460 cells (Wu et al., 2014). Ampelopsis grossedentata derived Dihydromyricetin is a flavonoid reported to induce DNA damage signaling modulator ATM in osteosarcoma cells. Moreover, p38MAPK and AMPK were also noted to be activated in Dihydromyricetin treated cells. More importantly, Dihydromyricetin exerted its effects via AMPK mediated negative regulation of GSK-3 $\beta$ in osteosarcoma cells (Zhao et al., 2014). Pseudolarix kaempferi Gordon derived Pseudolaric acid B is a diterpene acid recently been shown to induce G2/M arrest in HeLa cells via ATM mediated downstream signaling.

\section{Conclusion}

Overwhelmingly increasing list of natural and synthetic agents is currently being tested for efficacy as evidencd by in-vitro and in-vivo analysis. Methanolic extracts of Colchicum baytopiorum were reported to be effective against leukemic cell lines (Pirildar et al., 2010). Bioactive ingredients of Colchicum baytopiorum can further be tested to note if DNA damage signaling modulators are activated in treated cancer cell lines. Research over the years has added very important pieces of information to puzzle of DNA damage signaling and apoptosis. There is a progressive increase in the scientific evidence, deepening our understanding about crosstalk between ATM and autophagy and how autophagy inhibition improves synthetic and natural agents induced apoptosis in cancer cells. ATM is a master-regulator of wide ranging molecular mechanisms and improved knowledge regarding ATM biology will be helpful in maximizing therapeutic values of phytochemicals. Phytochemicals mediated regulation of DNA damage signaling is also providing detailed information about the complex molecular architecture. Phytochemicals mediated control of autophagic response and inhibitory effects on cancer progression are also being studied in xenografted mice. Low bioavailability of phytochemicals is another stumbling block that requires detailed research and it needs to be determined which strategy will be used (intravenous administration or oral intake) for evaluation of efficacy of phytochemicals in cancer models.

\section{References}

Bais MV, Ozdener GB, Sonenshein GE, et al (2014). Effects of tumor-suppressor lysyl oxidase propeptide on prostate cancer xenograft growth and its direct interactions with DNA repair pathways. Oncogene, [Epub ahead of print].

Borodkina A, Shatrova A, Abushik P, et al (2014). Interaction between ROS dependent DNA damage, mitochondria and p38 MAPK underlies senescence of human adult stem cells. Aging (Albany NY), 6, 481-95.

Carvalho S, Vítor AC, Sridhara SC, et al (2014). SETD2 is required for DNA double-strand break repair and activation of the p53-mediated checkpoint. Elife (Cambridge), 3, e02482.

Colis L, Peltonen K, Sirajuddin P, et al (2014). DNA intercalator BMH-21 inhibits RNA polymerase I independent of DNA damage response. Oncotarget, 5, 4361-9.

Dixit D, Ghildiyal R, Anto NP, et al (2014). Chaetocin-induced ROS-mediated apoptosis involves ATM-YAP1 axis and JNK-dependent inhibition of glucose metabolism. Cell Death Dis, 5, e1212.

Han D, Li SJ, Zhu YT, Liu L, Li MX (2013). LKB1/AMPK/ mTOR signaling pathway in non-small-cell lung cancer. Asian Pac J Cancer Prev, 14, 4033-9.

Lee JH, Guo Z, Myler LR, et al (2014). Direct activation of ATM by resveratrol under oxidizing conditions. PLoS One, 9, e97969.

Lee SH, Cheng H, Yuan Y, et al (2014). Regulation of ionizing radiation-induced adhesion of breast cancer cells to fibronectin by Alpha5beta1 integrin. Radiat Res, 181, 650-8.

Lim CB, Fu PY, Ky N, et al (2014). NF-kappaB p65 repression by the sesquiterpene lactone, Helenalin, contributes to the induction of autophagy cell death. BMC Complement Altern Med, 12, 93.

Lin CS, Wang YC, Huang JL, Hung CC, Chen JY (2012). Autophagy and reactive oxygen species modulate cytotoxicity induced by suppression of ATM kinase activity in head and 
Ammad Ahmad Farooqi et al neck cancer cells. Oral Oncol, 48, 1152-8.

Liu H, Zhou L, Shi S, et al (2014). Oligosaccharide G19 inhibits U-87 MG human glioma cells growth in vitro and in vivo by targeting epidermal growth factor (EGF) and activating p53/p21 signaling. Glycobiology, 24, 748-65.

Liu L, Yim H, Choi JH, et al (2014). ATM kinase promotes both caspase- 8 and caspase- 9 activation during TNF- $\alpha$-induced apoptosis of HeLa cells. FEBS Lett, 588, 929-35.

Luo GX, Cai J, Lin JZ, et al (2012). Autophagy inhibition promotes gambogic acid-induced suppression of growth and apoptosis in glioblastoma cells. Asian Pac J Cancer Prev, 13, 6211-6.

Luo X, Suzuki M, Ghandhi SA, et al (2014). ATM regulates insulin-like growth factor 1-secretory clusterin (IGF-1sCLU) expression that protects cells against senescence. PLoS One, 9, e99983.

Malbert-Colas L, Ponnuswamy A, Olivares-Illana V, et al (2014). HDMX Folds the Nascent p53 mRNA following Activation by the ATM Kinase. Mol Cell, 54, 500-11.

Mariño G, Niso-Santano M, Baehrecke EH, Kroemer G (2014). Self-consumption: the interplay of autophagy and apoptosis. Nat Rev Mol Cell Biol, 15, 81-94.

Pandey S, Chandravati (2012). Autophagy in cervical cancer: an emerging therapeutic target. Asian Pac J Cancer Prev, 13, 4867-71.

Pirildar S, Sütlüpınar N, Atasever B, et al (2010). Chemical constituents of the different parts of colchicum baytopiorum (Liliaceae) and their cytotoxic activities on K562 and HL60 cell-lines. Pharm Biol, 48, 32-9.

Sasca D, Hähnel PS, Szybinski J, et al (2014). SIRT1 prevents genotoxic stress-induced p53 activation in acute myeloid leukemia. Blood, 124, 121-33.

Shi M, Zhang T, Sun L, et al (2013). Calpain, Atg5 and Bak play important roles in the crosstalk between apoptosis and autophagy induced by influx of extracellular calcium. Apoptosis, 18, 435-51.

Sun CC, Xu HM, Yuan Y, et al (2014). Riccardin D, a macrocyclic bisbibenzy, inhibits human breast cancer growth through the suppression of telomerase activity. Basic Clin Pharmacol Toxicol, [Epub ahead of print].

Toulany M, Mihatsch J, Holler M, Chaachouay H, Rodemann HP. Cisplatin-mediated radiosensitization of non-small cell lung cancer cells is stimulated by ATM inhibition. Radiother Oncol, 111, 228-36.

Tripathi DN, Chowdhury R, Trudel LJ, et al (2013). Reactive nitrogen species regulate autophagy through ATM-AMPKTSC2-mediated suppression of mTORC1. Proc Natl Acad Sci USA, 110, E2950-7.

Wang FZ, Fei HR, Cui YJ, et al (2014). The checkpoint 1 kinase inhibitor LY2603618 induces cell cycle arrest, DNA damage response and autophagy in cancer cells. Apoptosis, 19, 1389-98.

Wu SH, Wu TY, Hsiao YT, et al (2014). Bufalin induces cell death in human lung cancer cells through disruption of DNA damage response pathways. Am J Chin Med, 42, 729-42.

Yan Y, Hein AL, Greer PM, et al (2014). A novel function of HER2/Neu in the activation of G2/M checkpoint in response to $\gamma$-irradiation. Oncogene, [Epub ahead of print].

Yang C, Hao J, Kong D, et al (2014). ATM-mediated Mad1 Serine 214 phosphorylation regulates Mad1 dimerization and the spindle assembly checkpoint. Carcinogenesis, [Epub ahead of print].

Yao G, Qi M, Ji X, et al (2014). ATM-p53 pathway causes G2/M arrest, but represses apoptosis in pseudolaric acid B-treated HeLa cells. Arch Biochem Biophys, 558, 51-60.

Yousefi S, Perozzo R, Schmid I, et al (2006). Calpain-mediated cleavage of Atg5 switches autophagy to apoptosis. Nat Cell
Biol, 8, 1124-32.

Zhang HQ, He B, Fang N, et al (2013). Autophagy inhibition sensitizes cisplatin cytotoxicity in human gastric cancer cell line SGC7901. Asian Pac J Cancer Prev, 14, 4685-8.

Zhang JW, Zhang SS, Song JR, et al (2014). Autophagy inhibition switches low-dose camptothecin-induced premature senescence to apoptosis in human colorectal cancer cells. Biochem Pharmacol, 90 (3), 265-75.

Zhao L, Chen H, Zhan YQ, et al (2014). Serine 249 phosphorylation by ATM protein kinase regulates hepatocyte nuclear factor- $1 \alpha$ transactivation. Biochim Biophys Acta, 1839 (7), 604-20.

Zhao Z, Yin JQ, Wu MS, et al (2014). Dihydromyricetin activates AMP-activated protein kinase and P38MAPK exerting antitumor potential in osteosarcoma. Cancer Prev Res (Phila), [Epub ahead of print].

Zou Y, Wang Q, Li B, Xie B, Wang W (2014). Temozolomide induces autophagy via ATM-AMPK-ULK1 pathways in glioma. Mol Med Rep, 10, 411-6. 